\title{
Vulnerability Analysis of Soft Caving Tunnel Support System and Surrounding Rock Optimal Control Technology Research
}

\author{
Ming Ji, ${ }^{1}$ Hongjun Guo, ${ }^{1}$ Yidong Zhang, ${ }^{2}$ Liang Cheng, ${ }^{1}$ and Yinlin Ji ${ }^{1}$ \\ ${ }^{1}$ School of Mines, Key Laboratory of Deep Coal Resource Mining, Ministry of Education of China, \\ China University of Mining \& Technology, Xuzhou 221116, China \\ ${ }^{2}$ State Key Laboratory of Coal Resources and Mine Safety, School of Mines, China University of Mining \& Technology, \\ Xuzhou 221116, China
}

Correspondence should be addressed to Yidong Zhang; ydzhang@cumt.edu.cn

Received 24 December 2013; Revised 29 March 2014; Accepted 31 March 2014; Published 27 April 2014

Academic Editor: Paulo Batista Gonçalves

Copyright (c) 2014 Ming Ji et al. This is an open access article distributed under the Creative Commons Attribution License, which permits unrestricted use, distribution, and reproduction in any medium, provided the original work is properly cited.

\begin{abstract}
The vulnerability assessment model, composed by 11 vulnerability factors, is established with the introduction of the concept of "vulnerability" into the assessment of tunnel support system. Analytic hierarchy process is utilized to divide these 11 factors into human attributes and natural attributes, and define the weight of these factors for the model. The "vulnerability" applied io the assessment of the tunnel support system model is reached. The vulnerability assessment model was used for evaluating and modifying the haulage tunnel \#3207 of Bo-fang mine panel \#2. The results decreased the vulnerability of the tunnel support system and demonstrated acceptable effects. Furthermore, the results show that the impact of human attributes on tunnel support systems is dramatic under the condition that natural attributes are permanent, and the "vulnerability" is exactly a notable factor to manifest the transformation during this process. The results also indicate that optimizing human attributes can attenuate vulnerability in tunnel support systems. As a result, enhancement of stability of tunnel support systems can be achieved.
\end{abstract}

\section{Introduction}

"Vulnerability" research began in the late 1970s and early 1980s. This concept was first proposed by Timmerman in 1981 [1]. Timmerman's research mainly focused on the field of geology, and his research appeared frequently in papers about risk and disaster $[2,3]$. With the growing concern on human safety and environmental impact, vulnerability was more frequently applied in evaluating the environmental changes and studying the relationship between environment and human development $[4,5]$. Vulnerability can be used to describe that relative systems and their components are susceptible to damage and lacking interference resistance and recovery capability. This concept is widely used in research on environmental aspects and nature disasters. Furthermore, in this field of research, vulnerability is often considered synonymous with sensitivity, fragility, and instability; however, these words have different meanings in various disciplines
[5-8]. Since its inception in 1981, the concept of vulnerability has been used in the research of the mining industry as well. For example, Wu et al. used this concept in the evaluation of water inrush in the floor of coal seams [9-11]. Su et al. and $\mathrm{Na}$ and Liu used it in the evaluation of economical and human systems in coal mine cities, respectively $[12,13]$. However, there is no previous research using the concept of vulnerability in the evaluation of supporting systems of loose and unstable roof systems for mining.

For research on support tunnels, Professor Dong mentioned the supporting theory of rock broken zone, which was based on large amounts of research and experimentation [14]. Based on the current research, Gou and Hou presented the theory of using bolts to strengthen the support for surrounding rocks [15]. This theory promoted the development of boltsupporting technology, as it was suitable for tunnel support in crushed rock. Afterward, many other new methods and theories have appeared, such as high strength and pretension 
TABLE 1: Quality ranks of rock mass integrity influence and quantitative scores.

\begin{tabular}{lccc}
\hline Structural surface spacing $(\mathrm{m})$ & Structural features & RQD $(\%)$ & Quality rank \\
\hline $1 \sim 2$ groups, $>1.0$ & Whole shape or thick-bedded & $>70$ & Integrity \\
$1 \sim 2$ groups, $>0.8$ & Massive or thick-bedded & $50 \leq R \leq 70$ & Relatively Integrity \\
$2 \sim 3$ groups, $>0.6$ & Block or in fissures, thin-bedded & $30 \leq R<50$ & Medium \\
$2 \sim 3$ groups, $>0.4$ & Massive fissure, fracture-like & $10 \leq R<30$ & Relatively broken \\
$3 \sim 4$ groups, $0.1 \sim 0.4$ & Granular form & $<10$ & Broken \\
\hline
\end{tabular}

TABLE 2: Quality ranks of rock strength and quantitative scores.

\begin{tabular}{|c|c|c|c|c|c|}
\hline \multirow{2}{*}{$\begin{array}{l}\text { Name } \\
\text { Uniaxial compressive strength }(\mathrm{MPa})\end{array}$} & \multicolumn{5}{|c|}{ Quantitative data and description } \\
\hline & $>80$ & $80 \leq S<60$ & $60 \leq S<40$ & $40 \leq S \leq 20$ & $<20$ \\
\hline Affecting the quality rank & Hard & Relatively hard & Medium & Relatively soft & Soft \\
\hline Scores & 2 & 4 & 6 & 8 & 10 \\
\hline
\end{tabular}

bolt-supporting technology, high prestressed and strong bolt support theories, and high prestressed bolt-supporting technology in coal tunnels [16-20]. These research efforts rarely involved tunnel supports under loose and unstable roof conditions; moreover, they do not consider the supporting bolts and the rock as a complete system to evaluate the effects of supports. Because soft caving tunnels have their own characteristics: the roof separates easily causing the roof to collapse, it is difficult to form an effective load-bearing structure; the large deformation of the tunnel's sides occurs easily, then spalling rib or the whole tunnel subsides easily [21]. Thus, the blot-supporting theory should be explored for adjustments to guide the bolt-supporting technology under the conditions loose roofs and roof which can easily fall. This paper addresses the evaluation indicators of vulnerability to consider the supporting bodies and rock as a whole objective and can be used for the design and optimization of bolt supporting in loose roofs and roof which can easily fall in tunnels.

\section{Analysis on Vulnerability}

2.1. Establishment of Evaluation Indicators. According to the analysis of the factors that affect the vulnerability of tunnel support systems, combining with the factors that affect the stability of tunnel surrounding rocks, the following indicators are the main factors that affect tunnel support systems. They not only indicate the main influences of geology but also reflect the effect of production technology from both nature and human characteristics. Moreover, these factors should be combined with the laws of motion for the tunnel's surrounding rock. Thus, the following factors are confirmed as evaluation indicators, using qualitative descriptions and quantitative analysis for evaluating the indicators' quantitative conversion.

(1) Integrity of Surrounding Rock. The surrounding rock's integrity relates to the number, shape, spacing, and roughness of surrounding rock's structural plane. The integrity of surrounding rock and the quantitative scores based on RQD are shown in Table 1.
(2) Roof Rock Strength. The strength of the roof rock can be indicated by roof rock's uniaxial compressive strength. Division rank and quantitative scores are shown in Table 2.

(3) Coal Strength. Coal strength is indicated by the coal's uniaxial compressive strength. The strength quality ranks and quantitative scores are shown in Table 3.

(4) Tunnel's Section Size. In similar geology conditions, the tunnel's section size determines the surrounding rocks stress distribution, size of loose circles, supporting range, and so on. The tunnel's section size quality ranks and quantitative scores are shown in Table 4.

(5) Depth of the Tunnel. The depth of the tunnel determines the gravity stress of the coal rock directly. It is one of the basic factors for the evaluation of the vulnerability of tunnel supporting systems. The ranks of the tunnel's depth and quantitative scores are shown in Table 5.

(6) Geological Structure. Geological structures affect the tunnel supporting system directly. Generally, folds, chasms, and collapse columns are three key factors that mainly affect a tunnel supporting system. According to the degree of folds, chasms, and collapse columns on a tunnel, the effect ranks and quantitative scores of geological structures are shown in Table 6 .

(7) Impact of Pillars. The width of pillar has a major impact on the tunnel supporting system's vulnerability. The ranks of the pillar width's impact and quantitative scores are shown in Table 7.

(8) Thickness of Strengthened Rock. Especially for layered, massive, and interactive structures that are composed of mudstone and sandstone, the thickness of the surrounding rock significantly contributes as an important indicator in tunnel supporting systems. It is shown that the thickness of the strengthened surrounding rock close to coal seams plays a key role in the tunnel supporting integrity. The quality ranks and quantitative scores are shown in Table 8. 
TABLE 3: Quality ranks of coal strength and quantitative scores.

\begin{tabular}{|c|c|c|c|c|c|}
\hline Name & & & ve data and de & & \\
\hline Uniaxial compressive strength $(\mathrm{MPa})$ & $>25$ & $25 \leq M<20$ & $20 \leq M<15$ & $15 \leq M \leq 10$ & $<10$ \\
\hline Affecting the quality rank & Hard & Relatively Hard & Medium & Relatively soft & Soft \\
\hline Scores & 2 & 4 & 6 & 8 & 10 \\
\hline
\end{tabular}

TABLE 4: Quality ranks of tunnel section size and quantitative scores.

\begin{tabular}{|c|c|c|c|c|c|}
\hline Name & & & ive data and d & & \\
\hline The size of tunnel's cross-section $\left(\mathrm{m}^{2}\right)$ & $<10$ & $10 \leq S<12$ & $12 \leq S<14$ & $14 \leq S \leq 16$ & $>16$ \\
\hline Affecting ranks & Small & Relatively small & Medium & Relatively large & Large \\
\hline Scores & 2 & 4 & 6 & 8 & 10 \\
\hline
\end{tabular}

TABLE 5: Quality ranks of tunnel buried depth and quantitative scores.

\begin{tabular}{lccccc}
\hline Name & \multicolumn{4}{c}{ Quantitative data and description } \\
\hline Depth $(\mathrm{m})$ & $0<D<100$ & $100 \leq D<300$ & $300 \leq D<500$ & $500 \leq D \leq 700$ & $D>700$ \\
Quality ranks & Shallow & Relatively shallow & Medium & Relatively deep & Deep \\
Scores & 2 & 4 & 6 & 8 & 10 \\
\hline
\end{tabular}

TABLE 6: Quality ranks of geological structure influence and quantitative scores.

\begin{tabular}{lccccc}
\hline Name & & \multicolumn{3}{c}{ Quantitative data and description } \\
\hline $\begin{array}{l}\text { Effect factors } \\
\text { and extent }\end{array}$ & $\begin{array}{c}\text { Single factor } \\
\text { (small) }\end{array}$ & $\begin{array}{c}\text { Double factors (small), } \\
\text { single factor (medium) }\end{array}$ & $\begin{array}{c}\text { Double factors (one medium } \\
\text { and one small), three (one } \\
\text { medium and two small) }\end{array}$ & $\begin{array}{c}\text { Single factor (large), double } \\
\text { factors (two medium), three } \\
\text { factors (two medium and one } \\
\text { small) }\end{array}$ & Others \\
Quality ranks & I & II & III & IV & V \\
Scores & 2 & 4 & 6 & V \\
\hline
\end{tabular}

TABLE 7: Quality ranks of coal pillar influence and quantitative scores.

\begin{tabular}{lccccc}
\hline Name & \multicolumn{4}{c}{ Quantitative data and description } \\
\hline Width of pillars $(\mathrm{m})$ & $>46$ & $26 \sim 45$ & $15 \sim 25$ & $7 \sim 14$ & $<6$ \\
Affecting ranks & Slight & General & Medium & Serious & Very serious \\
Scores & 2 & 4 & 6 & 8 & 10 \\
\hline
\end{tabular}

TABLE 8: Quality ranks of strengthened rock thickness and quantitative scores.

\begin{tabular}{|c|c|c|c|c|c|}
\hline Name & & & ve data and & & \\
\hline Strengthened rock thickness $(T / \mathrm{m})$ & $<2$ & $2 \leq T<4$ & $4 \leq T<6$ & $6 \leq T \leq 8$ & $>8$ \\
\hline Quality ranks & Small & Relatively small & Medium & Relatively large & Large \\
\hline Scores & 2 & 4 & 6 & 8 & 10 \\
\hline
\end{tabular}

TABLE 9: Quality ranks of support form and quantitative scores.

\begin{tabular}{lcccc}
\hline Name & \multicolumn{3}{c}{ Quantitative data and description } \\
\hline Supporting strength $(\mathrm{MPa})$ & $<0.1$ & $0.1 \sim 0.2$ & $0.2 \sim 0.3$ & $0.3 \sim 0.5$ \\
Affecting degree & Small & Relatively small & Medium & Relatively large \\
Scores & 2 & 4 & 6 & Large \\
\hline
\end{tabular}

TABLE 10: Quality ranks of tunnel repair and quantitative scores.

\begin{tabular}{lcccc}
\hline Name & \multicolumn{4}{c}{ Quantitative data and description } \\
\hline Repair rate $(R / \%)$ & $<10$ & $10 \leq R<20$ & $20 \leq R<30$ & $30 \leq R \leq 50$ \\
Affecting ranks & Small & Relatively small & Medium & Relatively large \\
Scores & 2 & 4 & 6 & 8 \\
\hline
\end{tabular}




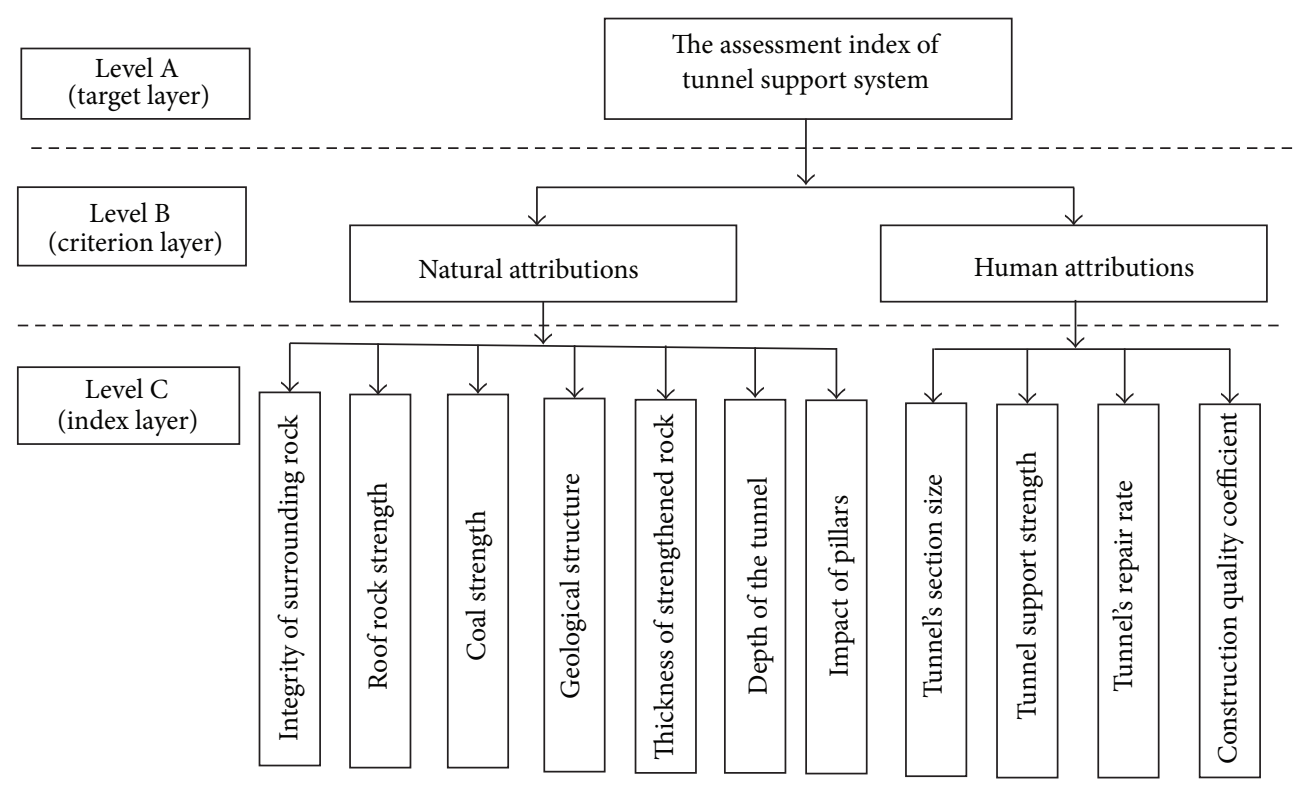

FIGURE 1: Hierarchical graph of vulnerability assessment index.

TABLE 11: Quality ranks of construction quality and quantitative scores.

\begin{tabular}{|c|c|c|c|c|c|}
\hline Name & & & ive data and $\mathrm{d}$ & & \\
\hline Construction quality coefficient (Q/\%) & $<10$ & $10 \leq Q<20$ & $20 \leq Q<30$ & $30 \leq Q \leq 50$ & $>50$ \\
\hline Affecting ranks & Small & Relatively small & Medium & Relatively large & Large \\
\hline Scores & 2 & 4 & 6 & 8 & 10 \\
\hline
\end{tabular}

(9) Tunnel Support Strength. The tunnel support strength is one of the most important factors in evaluating a tunnel supporting system. It includes many related factors, such as basic supporting methods and supporting diameters, and serves as a comprehensive indicator to evaluate supporting systems. Moreover, this indicator has high operability to measure the overall evaluation indicator. The quality ranks of support form and quantitative scores are shown in Table 9.

(10) Tunnel's Repair Rate. For a tunnel support evaluation, a tunnel repair should control the deformation of surrounding rock with one-time supported and avoid any need for multiple repairs. The tunnel repair should be evaluated by the principles of bolt supporting. The most effective way to achieve the tunnel repairs is to support the surrounding rock by bolts in conjunction with tunneling operations. However, the efforts to achieve the required structural support will be affected dramatically if using bolts to support the surrounding rock after the separation and damage of rock has occurred. Therefore, repair work of tunnels has a huge impact on the vulnerability of tunnel supporting systems. The quality ranks of the tunnel's repair rate and quantitative scores are shown in Table 10.

(11) Construction Quality Coefficient. The quality of construction impacts the vulnerability of tunnel' supporting systems directly. It is one of the human impact factors. A tunnel's natural properties were focused on more during previous related evaluations. In fact, the construction quality is very important for the vulnerability of tunnel supporting systems. The direct manifestation of the construction quality in tunnel supporting systems is to monitor the construction quality of bolts. Evaluation of the bolting quality shall be conducted by the monitoring of the bolts in three sections: the bolts' drawing force, the bolts' preload, and the diameter of the bolt supports (spacing, angle, and exposed length). The coefficient of construction quality is the sum of the failure rates of these three sections. Quality ranks of construction quality and quantitative scores are shown in Table 11.

Overall, as shown in Figure 1, the above eleven indicators can evaluate the vulnerability of tunnel supporting systems from natural factors and human factors.

2.2. Construction of Basic Model. The weighted average method is used to calculate the vulnerability evaluation values of the tunnel supporting system. The basic model is made as follows:

$$
Y_{j}=\sum_{I=1}^{n} c_{i j} w_{i j},
$$

where $c_{i j}$ is the evaluation score of $i$ th factor in the $j$ th tunnel and varied from 2 to $4,6,8$, and 10 in the paper and $c_{i j}$ is the evaluation score of each factor in Tables 1 to 11 .

$w_{i j}$ is $j$ weighted value of $i$ th factor in the $j$ th tunnel; the value extent is $(0,1)$. 
TABLE 12: Grading standards of vulnerability of tunnel support system.

\begin{tabular}{lccccc}
\hline Value range of $Y_{j}$ & $Y_{j}<5$ & $5 \leq Y_{j}<5.5$ & $5.5 \leq Y_{j}<6.5$ & $6.5 \leq Y_{j} \leq 7$ & $7<Y_{j}$ \\
\hline Vulnerability & Small & Relatively small & Medium & Relatively large & Large \\
\hline
\end{tabular}

TABLE 13: Tag matrix.

\begin{tabular}{lcclc}
\hline Condition B & $C 1$ & $C 2$ & $\cdots$ & $C n$ \\
\hline$C_{1}$ & $a_{11}$ & $a_{12}$ & $\cdots$ & $a_{1 n}$ \\
$C_{2}$ & $a_{21}$ & $a_{22}$ & $\ldots$ & $a_{2 n}$ \\
$\vdots$ & $\vdots$ & $\vdots$ & $\ldots$ & $\vdots$ \\
$C_{n}$ & $a_{n 1}$ & $a_{n 2}$ & & $a_{n n}$ \\
\hline
\end{tabular}

TABLE 14: 1-9 scaling method.

\begin{tabular}{lc}
\hline The ratio of factors & Quantized values \\
\hline Equally important & 1 \\
Somewhat important & 3 \\
More important & 5 \\
Highly important & 7 \\
Extremely important & 9 \\
Intermediate value between & $2,4,6,8$ \\
two adjacent judgments & \\
\hline
\end{tabular}

TABLE 15: Value of RI.

\begin{tabular}{|c|c|c|c|c|c|c|c|c|c|c|c|}
\hline Matrix order & 1 & 2 & 3 & 4 & 5 & 6 & 7 & 8 & 9 & 10 & 11 \\
\hline RI & 0 & 0 & 0.58 & 0.90 & 1.12 & 1.24 & 1.32 & 1.41 & 1.45 & 1.49 & 1.51 \\
\hline
\end{tabular}

According to the value of $Y_{j}$ (vulnerability), the vulnerability of a tunnel supporting system can be divided into five ranks, as shown in Table 12.

The confirmation of weighted values is one of the most important factors in determining whether the model is reasonable. The reasonable weight determination should be done for every factor, since every factor has a different impact on the vulnerability of tunnel supporting systems. Currently, there are many mathematical methods that can be used to confirm the weighted value rate of any given factor. According to the feature of this model, this paper uses analytic hierarchy process (AHP) to confirm the weighted value. As shown in Figure 1, this paper divides the evaluation factors into three layers: target layer, criterion layer, and index layer. Every two layers' factors are compared as well, to judge the importance of every factor and confirm their values. Moreover, the consistency of the comparison matrix is evaluated to confirm the weighted value of the evaluate indicator.

AHP model was used in the following steps [22, 23].

Step 1. Establish the hierarchical structure model. Distribute question's targets and schemes rationally according to the demand of decision objective, and confirm the components of every layer's factors.

Step 2. Establish the comparison matrix. From the second layer of the hierarchical model, for every factor in the same layer which belongs to last layer's factors, a comparison matrix was established by using the comparison method and 1-9 scaling method (shown in Table 14). For example, for a layer's indicator $\mathrm{B}$ with factors $C_{1}, C_{2}, C_{3}, \ldots, C_{n}$, which belong to $\mathrm{B}$, evaluate indicator $\mathrm{B}$ as the evaluation target to pairwise comparison. The comparison matrix is shown in Table 13.

In the comparison matrix, the factor $a_{i j}$ is the ratio of the degree of importance for $C_{i}$ and $C_{j}$ in condition $\mathrm{B}$ :

$$
a_{i j}=\frac{A_{i}}{A_{j}}
$$

where $A_{i}$ is the importance of factor $C_{i}$ in condition B and $A_{j}$ is the importance of factor $C_{j}$ in condition $B$.

A comparison matrix can be generated by comparing any two factors $C_{i}$ and $C_{j}$ as the matrix structure follows the 1-9 scale method, as shown in Table 14.

Step 3. Calculation of weighted vector and the biggest nonzero feature root of the comparison matrix: the weighted vector is the feature component which corresponds to the characteristic root of the matrix.

The calculation process is as follows:

$$
\begin{gathered}
\widetilde{w}_{i j}=\frac{a_{i j}}{\sum_{i=1}^{n} a_{i j}}, \quad j=1,2, \ldots, n, \\
\widetilde{w}_{i}=\sum_{j=1}^{n} \widetilde{w}_{i j}, \\
w_{i}=\frac{\widetilde{w}_{i}}{\sum_{j=1}^{n} \widetilde{w}_{i j}}, \\
\lambda_{\max }=\frac{1}{n} \sum_{j=1}^{n} \frac{(A w)_{i}}{w_{i}},
\end{gathered}
$$

where $A$ is the comparison matrix; $a_{i j}$ is each element in $A$; $\widetilde{w}_{i j}$ and $\widetilde{w}_{i}$ are the intermediate variables in the calculation and $w_{i}$ is the weight of each factors; $w$ is the weighted vector and $w=\left(w_{1}, w_{2}, \ldots, w_{n}\right)^{T} ; \lambda_{\max }$ is the biggest nonzero feature root of $A$.

Step 4. Consistency test of pairwise comparison matrix: because $a_{i j}$ is an approximation of subjective evaluation, it might cause a calculated deviation of the comparison matrix's eigenvalues if there is a deviation of judgment. Thus, a consistency test is necessary. The indicator CI can be calculated from

$$
\mathrm{CI}=\frac{\lambda_{\max }-n}{n-1} .
$$

A random subjective evaluation might cause the consistency deviation; thus, CI should also be compared with the 


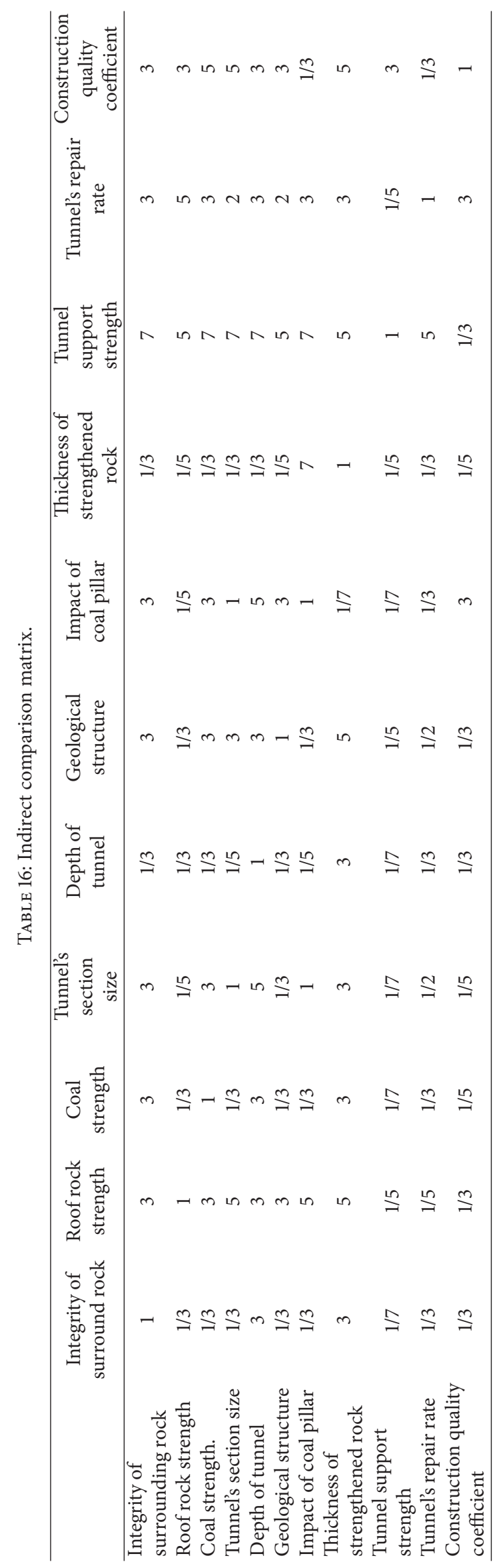




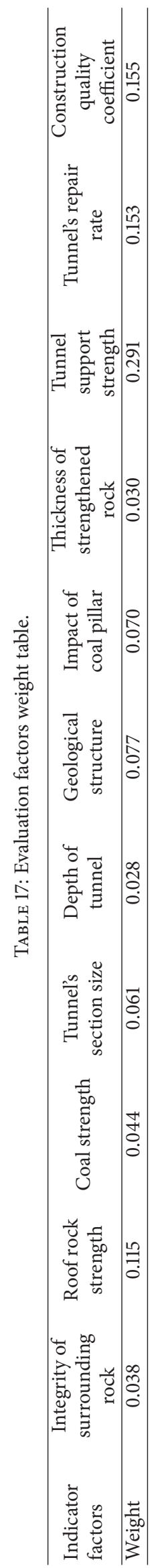


TABLE 18: Summary of the vulnerability of mine tunnel.

\begin{tabular}{|c|c|c|c|c|c|c|c|c|c|c|c|c|c|c|}
\hline & & \multicolumn{11}{|c|}{ Vulnerability indicators of tunnel's supporting systems } & \multicolumn{2}{|r|}{ Vulnerability } \\
\hline & & A & $\mathrm{B}$ & $\mathrm{C}$ & $\mathrm{D}$ & $\mathrm{E}$ & $\mathrm{F}$ & G & $\mathrm{H}$ & I & $\mathrm{J}$ & $\mathrm{K}$ & $Y_{j}$ & Vulnerability grade \\
\hline \multirow{8}{*}{ Mine name } & Xian Dewang mine & 30.57 & 25.37 & 5.45 & 12.6 & 460 & II & 5 & 7.33 & 0.361 & 27 & 31 & 6.628 & Higher \\
\hline & Gu Shuyuan mine & 75.6 & 105.35 & 17.79 & 11.25 & 210 & I & 30 & 0.7 & 0.036 & 2 & 8 & 4.946 & Lower \\
\hline & Yang Quan 1\#mine & 44 & 33 & 15.4 & 10.5 & 370 & II & $\geq 50$ & 5.27 & 0.403 & 15 & 25 & 5.158 & Lower \\
\hline & Xia Gou mine & 37 & 30 & 16.9 & 10.3 & 350 & I & 10 & 10 & 0.194 & 23 & 36 & 7.464 & Highest \\
\hline & Chang Cun mine & 12 & 51.3 & 13.3 & 14.07 & 400 & II & $\geq 50$ & 7.15 & 0.438 & 8 & 19 & 4.78 & Lower \\
\hline & Si He mine & 68 & 75.56 & 21.9 & 19.25 & 400 & IV & 15 & 5.3 & 0.289 & 9 & 8 & 5.144 & Lower \\
\hline & Dong Tan mine & 28 & 38 & 17.5 & 14.5 & 560 & II & 2 & 6.05 & 0.308 & 17 & 15 & 5.564 & Moderate \\
\hline & Xiao Kang mine & 8 & 17.6 & 14.2 & 10.2 & 525 & II & $\geq 50$ & 5.4 & 0.356 & 70 & 35 & 6.912 & Higher \\
\hline
\end{tabular}

A: integrity of surrounding rock; B: roof rock strength; C: coal strength; D: tunnel's section size; E: depth of tunnel; F: geological structure; G: impact of coal pillar; H: thickness of strengthened rock; I: tunnel support strength; J: tunnel's repair rate; K: construction quality coefficient.

average random consistency indicator RI. The value of RI is shown in Table 15 [24]. During testing of the comparison matrix the consistency rate $\mathrm{CR}$ can be calculated in (8)

$$
\mathrm{CR}=\frac{\mathrm{CI}}{\mathrm{RI}}
$$

The values of $\mathrm{CI}$ and $\mathrm{CR}$ must both be less than 0.1 [22-25].

2.3. The Confirmation of Indicator Weights. The vulnerability matrix, with eleven vulnerability indicators of tunnel supporting systems based on AHP, is constructed by using 1-9 scale method and shown in Table 16.

2.4. Consistency Test. The biggest feature root of the comparative matrix was calculated to be $\lambda_{\max }=11.851$. Therefore, following (7) above,

$$
\mathrm{CI}=\frac{11.851-11}{11-1}=0.0851<0.1 .
$$

The consistency indicator CI meets the requirements as defined above. Next, evaluate the consistency rate CR, taking into account the calculated value for CI, by (8):

$$
\mathrm{CR}=\frac{0.0851}{1.51}=0.0564<0.1
$$

The consistency rate CR meets the requirements. Thus, the comparison matrix meets the consistency requirements.

2.5. Vulnerability Evaluation. The weight of evaluation indicators for the vulnerability of the tunnel supporting systems is calculated by using (3)-(5) and shown in Table 17.

The value of a tunnel supporting system's vulnerability evaluation can be calculated according to the evaluation factors weight in Table 17 and (1).

According to the calculation of this model, the evaluation factor scores coming from the tunnel's exact parameters, and the vulnerability ranks in Table 12 , the ranks of tunnels supporting system's vulnerability can be confirmed. Table 18 shows the vulnerability index of various mines.

\section{Application Examples}

In panel $2^{\#}$ of the Bo-fang mine, the floor rock was falling with the excavating of this tunnel and the temporary supporting system was ineffective. These issues were not only affecting the excavation speed but also illustrated many security risks in the floor's structure. There was serious deformation and destruction of the tunnel's rock and supporting body. Based on field geological data, operating procedures, and the standards founded above, the influence scores of 3203 haulage tunnel supporting system vulnerability evaluation factors can be confirmed, as shown in Table 19.

Taking the systems evaluation scores into consideration, the evaluation model of tunnel supporting system's vulnerability is calculated at $Y_{j}=6.638$.

According to the ranking standards of tunnel supporting system vulnerability model in Table 12 , a vulnerability index of $Y_{j}=6.638$ indicates that the supporting system's vulnerability is relatively large. Based on indicator factors that impact the vulnerability, the tunnel's nature factors are constant. However, for human factor, the tunnel's size could not become smaller because of production needs. Thus, the primary solutions to improve the vulnerability index of the tunnels supporting system are to raise the tunnel supporting strength, decrease the construction quality factors, guarantee supporting disposable, and decrease the repair rate. According to previous calculation, the vulnerability $Y_{j}$ should be lower than 5.5 .

The supporting system was optimized using the above analysis in haulage tunnel 3207 , which contained a loose caving roof, also found in panel $2^{\#}$, by surrounding rock. After analysis for optimization, there are three main changes.

(1) Increase of tunnel supporting strength: according to theoretical analysis and simulation to optimize the supporting parameters of mining tunnel in panel $2^{\#}$, the density of bolt supporting was increased. All longitudinal reinforcement bolt materials were changed from BHRB335 to BHRB500. The breaking load of the bolt body was improved. The bolts can now control the surrounding rock's deformation effectively and reduce the previous phenomenon of breaking bolts. The anchor arrangement was optimized from a layout 


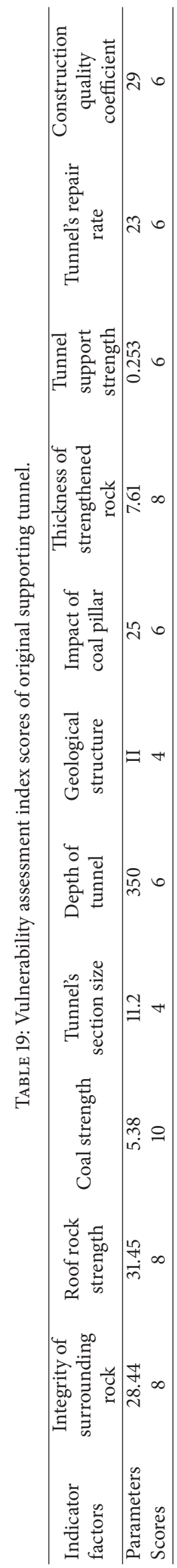




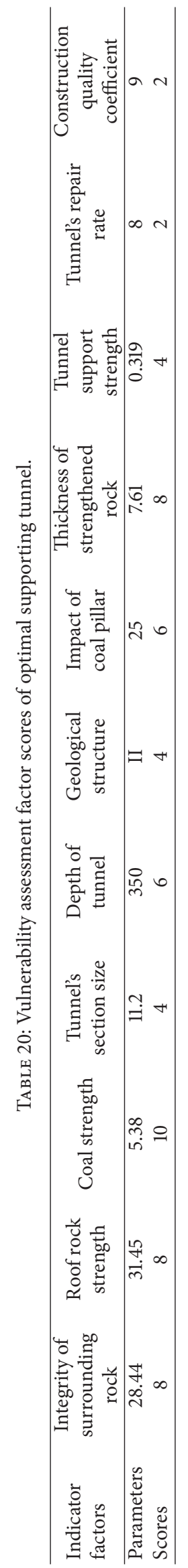


of one anchor in every row to a streaky layout. These changes improved the overall supporting strength by $26.2 \%$.

(2) Change of construction quality factor: because of the many problems occurring in haulage tunnel 3203, such as breaking bolts and anchors, as well as a falling roof, the higher quality requirements and standards for supporting and excavation were put forward. The technical training of workers was organized. Afterwards, pullout force, preload force, and supporting geometric parameters were monitored for bolting after excavation of the tunnel. The failure rate of bolting working quality factors was reduced to less than $20 \%$.

(3) Repair rate influence: because of the improvement of the tunnel's excavation and supporting quality, the success rate of the tunnel's initial support system has a dramatic increase. Thus, the repair rate has reduced. The repair rate of haulage tunnel 3207 reduced to $21 \%$ lower than the repair rate of haulage tunnel 3203.

The influence factor scores in haulage tunnel 3207 supporting system's vulnerability evaluation are shown in Table 20.

Taking these scores into the evaluation model of supporting system, the vulnerability index was determined to be $Y_{j}=4.964$.

According to Table 12, after the optimization plan of the tunnel supporting system was implemented, the vulnerability $Y_{j}$ is 4.964. This indicates that the vulnerability of this system is small and meets the requirement that the vulnerability $Y_{j}$ be less than 5.5. Afterward, through field monitoring and data analysis of the supporting and mine pressure observations, optimizing the supporting scheme achieved improved technical and economical results.

\section{Conclusions}

(1) According to the evaluation model of vulnerability indicators, each indicator weight can be calculated using AHP. Human properties have a significant influence on a tunnel supporting system where the natural properties indicators remain constant. Moreover, vulnerability is a key indicator which illustrates this change.

(2) Through engineering applications, the full enhancement of each human property in the vulnerability evaluation model, such as supporting strength, construction quality factor, and repair rate, can reduce the vulnerability of the tunnel's system effectively while enhancing the tunnel's system stability given similar geological and mining conditions. Furthermore, such improvements result in desirable economical benefits.

(3) As the coal mining scale gradually increases in China, a unified evaluation standard for tunnel supporting systems is offered in utilization of the vulnerability index model to fully consider each contributing indicator's effects. Standardization of this model as an evaluation tool will result in the overall improvement of tunnel support systems throughout the industry. However, this model methodology is still an incipient concept in the evaluation of tunnel support systems and requires further research.

\section{Conflict of Interests}

The authors declare that there is no conflict of interests regarding the publication of this paper.

\section{Acknowledgments}

This paper is supported by the Natural Science Foundation of Jiangsu Province, China (Grant no. BK20130189); the Priority Academic Program Development of Jiangsu Higher Education Institutions; the Fundamental Research Funds for the Central Universities (2011QNA12); and the Postdoctoral Foundation of Jiangsu Province (1101033C) and Funded by the Open Projects of State Key Laboratory of Coal Resources and Safe Mining, CUMT (SKLCRSM12X05).

\section{References}

[1] P. Timmerman, Vulnerability Resilience and the Collapse of Society: A Review of Models and Possible Climatic Applications, Institute for Environmental Studies, University of Toronto, Toronto, Canada, 1981.

[2] A. Maffei, S. Martino, and A. Prestininzi, "From the geological to the numerical model in the analysis of gravity-induced slope deformations: an example from the Central Apennines (Italy)," Engineering Geology, vol. 78, no. 3-4, pp. 215-236, 2005.

[3] A. C. Waltham and G. M. Swift, "Bearing capacity of rock over mined cavities in Nottingham," Engineering Geology, vol. 75, no. 1, pp. 15-31, 2004.

[4] S. R. Zhu, Vulnerability Assessment Model and Application in Coal Enterprise [M.S. thesis], Henan University, 2008.

[5] Y. H. Liu and X. B. Li, Fragile Ecological Environment and Sustainable Development, The Commercial Press, Beijing, China, 2001.

[6] Y. C. Shen and Y. T. Zhang, The Reason of Fragile Ecological Environment's Formation and Evolution, and Its Regional Differentiation, the Technology and Research on Ecological Environment Integrated Renovation in China, Science and Technology Press, Beijing, China, 1992.

[7] J. Y. Shen, To Investigate the Method of Sensitivity Evaluation of Fragile Environment, the Technology and Research on Ecological Environment Integrated Renovation, Science and Technology Press, Beijing, China, 1995.

[8] J. Yang, Z. G. Tao, B. L. Li et al., "Stability assessment and feature analysis of slope in Nanfen Open Pit Iron Mine," International Journal of Mining Science and Technology, vol. 22, no. 3, pp. 329333, 2012.

[9] Q. Wu, Z.-L. Zhang, S.-Y. Zhang, and J.-F. Ma, "New practical methodology of the coal floor water bursting evaluating II: the vulnerable index method," Journal of the China Coal Society, vol. 32, no. 11, pp. 1121-1126, 2007.

[10] Q. Wu, S.-H. Xie, Z.-J. Pei, and J.-F. Ma, "New practical methodology of the coal floor water bursting evaluating III: the application of ANN vulnerable index method based on GIS," 
Journal of the China Coal Society, vol. 32, no. 12, pp. 1301-1306, 2007.

[11] Q. Wu, W. Pang, and Y.-C. Dai, "Vulnerability forecasting model based on coupling technique of GIS and ANN in floor groundwater bursting," Journal of the China Coal Society, vol. 31, no. 3, pp. 314-319, 2006.

[12] F. Su, P. Y. Zhang, and H. Li, "Vulnerability assessment of coal mining cities economic systems in China," Geographical Research, vol. 27, no. 4, pp. 907-916, 2008.

[13] W. Na and J. S. Liu, "Vulnerability and its evaluation system of human land system in mining city," City Problems, no. 144, pp. 43-48, 2007.

[14] F. T. Dong, Design and Construction of Tunnel, China University of Mining and Technology Press, Xuzhou, China, 1994.

[15] P. F. Gou and C. J. Hou, "Experimental study on strengthening the strength of surrounding rock," Journal of Chongqing University, vol. 23, no. 3, pp. 35-39, 2000.

[16] X. Q. Guo, X. B. Mao, C. Ma, and J. L. Huang, "Bolt support mechanism based on elastic theory," International Journal of Mining Science and Technology, vol. 23, no. 4, pp. 469-474, 2013.

[17] B. Meng, H. W. Jing, K. F. Chen, and H. J. Su, "Failure mechanism and stability control of a large section of very soft roadway surrounding rock shear slip," International Journal of Mining Science and Technology, vol. 23, no. 1, pp. 127-134, 2013.

[18] S. Yan, J. B. Bai, W. F. Li, J. G. Cheng, and L. Li, "Deformation mechanism and stability control of roadway along a fault subjected to mining," International Journal of Mining Science and Technology, vol. 22, no. 4, pp. 559-565, 2012.

[19] H.-P. Kang, J. Lin, and Y.-Z. Wu, "High pretensioned stress and intensive cable bolting technology set in full section and application in entry affected by dynamic pressure," Journal of the China Coal Society, vol. 34, no. 9, pp. 1153-1159, 2009.

[20] N. Zhang and M.-S. Gao, "High-strength and pretension bolting support of coal roadway and its application," Journal of China University of Mining and Technology, vol. 33, no. 5, pp. 524-527, 2004.

[21] J. B. Bai, C. J. Hou, M. Du, and D. Ma, "On bolting support of roadway in extremely soft seam of coal mine with complex roof," Chinese Journal of Rock Mechanics and Engineering, vol. 20, no. 1, pp. 53-56, 2001.

[22] W. Pedrycz and M. Song, "A granulation of linguistic information in AHP decision-making problems," Information Fusion, vol. 17, pp. 93-101, 2014.

[23] Y. C. Chen, T. H. Yu, P. L. Tsui, and C. S. Lee, "A fuzzy AHP approach to construct international hotel spa atmosphere evaluation model," Quality \& Quantity, vol. 48, pp. 645-657, 2014.

[24] T. L. Saaty, The Analytic Hierarchy Process, McGraw-Hill, New York, NY, USA, 1980.

[25] S. Chakraborty and D. Banik, "Design of a material handling equipment selection model using analytic hierarchy process," International Journal of Advanced Manufacturing Technology, vol. 28, no. 11-12, pp. 1237-1245, 2006. 


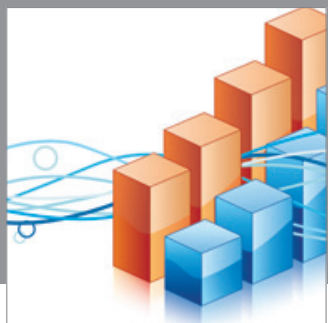

Advances in

Operations Research

mansans

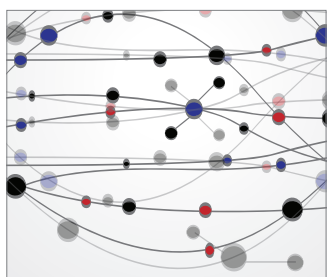

The Scientific World Journal
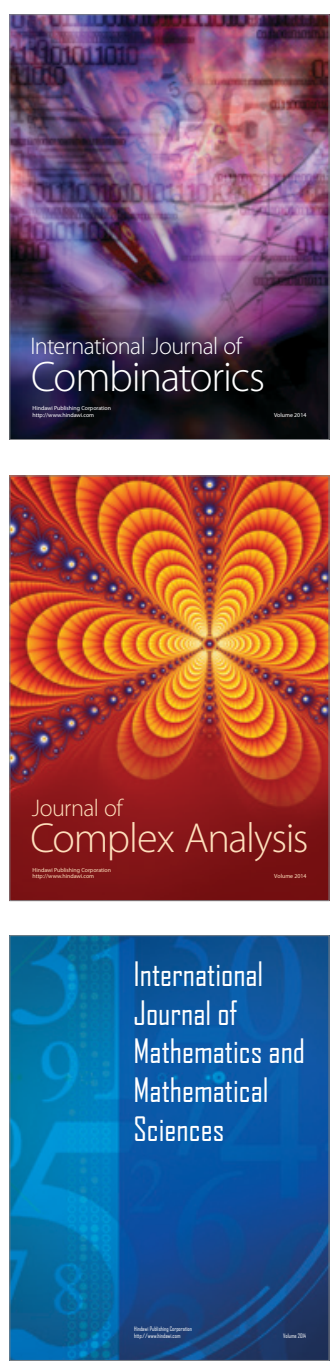
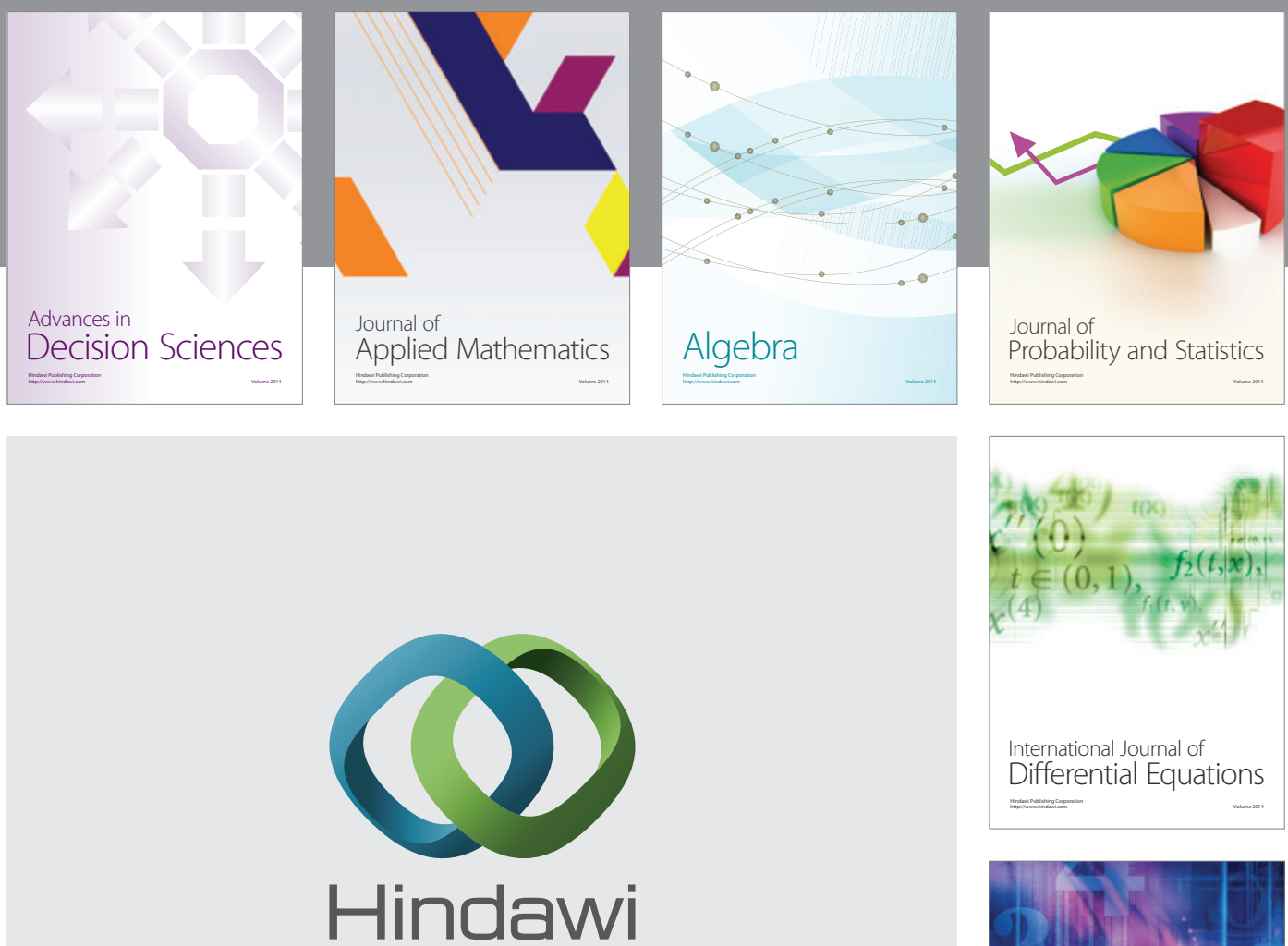

Submit your manuscripts at http://www.hindawi.com
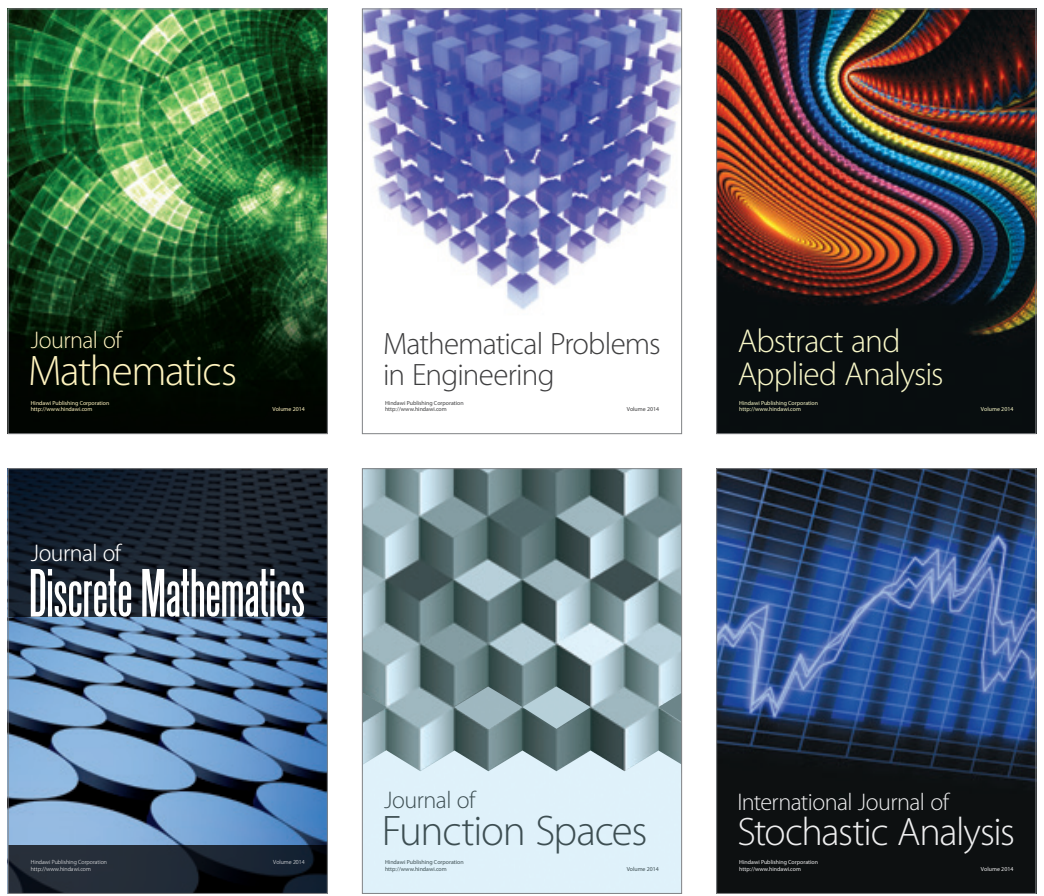

Journal of

Function Spaces

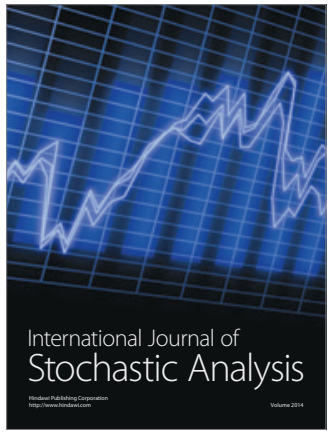

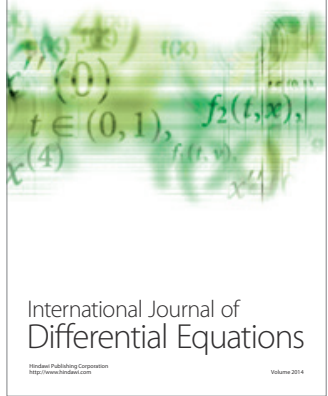
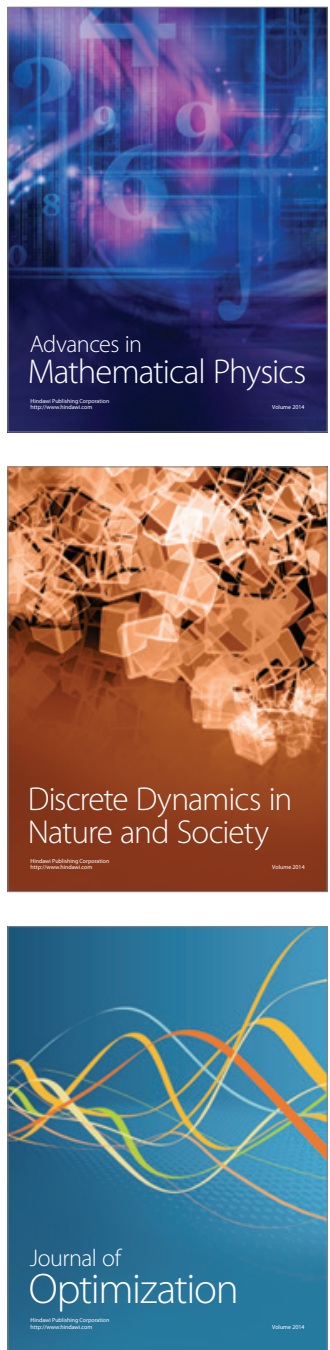